Check for updates

Cite this: RSC Adv., 2019, 9, 26598

\title{
Polyethylenimine-alginate nanocomposites based bone morphogenetic protein 2 gene-activated matrix for alveolar bone regeneration $\dagger$
}

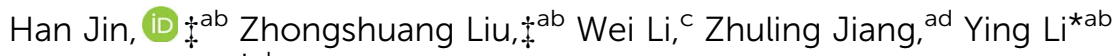 \\ and Bin Zhang*ab
}

The repair and treatment of lost or damaged alveolar bone is of great significance in dentistry. Geneactivated matrix (GAM) technology provides a new way for bone regeneration. It is a local gene delivery system, which can not only recruit cells, but also influence their fate. For this purpose, we fabricated a bone morphogenetic protein 2 (BMP-2) gene-loaded absorbable gelatin sponge (AGS) and studied its effect on promoting alveolar bone formation and preventing resorption following tooth extraction in rats. In order to obtain better transfection efficiency, polyethylenimine-alginate (PEI-al) nanocomposites were synthesized and used as gene vectors to deliver BMP-2 cDNA plasmids (PEI-al/pBMP-2). The transfection efficiency, BMP-2 protein expression and osteogenic differentiation of the cells were investigated in vitro. In vivo, we established an alveolar bone regeneration model by extracting the rats' left mandibular incisors. The rats were randomly assigned into 3 groups: control group, unfilled sockets; AGS group, sockets filled with PEI-al solution-loaded gelatin sponges; AGS/BMP group, sockets filled with PEI-al/pBMP-2 solution-loaded gelatin sponge. Radiological and histological assays were performed at 4 and 8 weeks later. In vitro transfection assays indicated that PEI-al/pBMP-2 complexes could effectively transfect MC3T3-E1 cells, promoting the secretion of BMP-2 protein for at least 14 days, as well as increasing the expression of osteogenesis-related gene, ALP activity and calcium deposition. In vivo, western blot analysis showed BMP-2 protein was expressed in bone tissues of AGS/BMP group. The relative height of the residual alveolar ridge and bone mineral density (BMD) of the AGS/BMP group were significantly greater than those in the AGS and control groups at 4 and 8 weeks, respectively. Histological examination showed that, at 4 weeks, osteoblasts had grown in a cubic shape around the new bone in the AGS/BMP group, suggesting new bone formation. In conclusion, the combination of PEI-al/pBMP-2 complexes and gelatin sponge could promote alveolar bone regeneration, which may provide an easy and valuable method for alveolar ridge preservation and augmentation.

Received 8th July 2019

Accepted 18th August 2019

DOI: $10.1039 / c 9 r a 05164 c$

rsc.li/rsc-advances

\section{Introduction}

Acquired injury, congenital disorders, tumors, periodontal disease and tooth loss often cause alveolar bone defects. These defects make it difficult to obtain adequate bone volume for the placement of dental implants or dentures; ${ }^{1}$ therefore, studies often focus on materials and procedures capable of reducing

\footnotetext{
anstitute of Hard Tissue Development and Regeneration, The Second Affiliated Hospital of Harbin Medical University, 246 Xuefu Road, Harbin, Heilongjiang 150001, China.E-mail: liying@hrbmu.edu.cn; zhangbin@hrbmu.edu.cn ${ }^{b}$ Heilongjiang Academy of Medical Sciences, Harbin, Heilongjiang 150001, China 'Department of Stomatology, Harbin Children's Hospital, Harbin, Heilongjiang 150001, China

${ }^{d}$ Department of Implantology, The Second Affiliated Hospital of Harbin Medical University, Harbin, Heilongjiang 150001, China

$\dagger$ Electronic supplementary information (ESI) available. See DOI: 10.1039/c9ra05164c

\$ Han Jin and Zhongshuang Liu contributed equally to this work.
}

bleeding and promoting effective bone regeneration, in order to prevent alveolar bone loss and keep the morphology and quality of the alveolar bone..$^{-4}$

Gelatin sponges have been used as hemostatic agents and wound coverings for a long time..$^{5,6}$ A previous study observed new bone formation in the maxillary sinus using only gelatin sponges as the graft material, which may provide adequate bone mass for implant placement. ${ }^{7}$ Since gelatin sponges have excellent biocompatibility, degradation into physiological endproducts $^{8-10}$ and suitable interaction with cells and other macromolecules, ${ }^{11-14}$ they have received increasing attention as scaffolds in the emerging field of tissue engineering. ${ }^{15-17}$

Bone morphogenetic proteins (BMPs) are potent growth factors for bone formation and remodeling, due to their powerful osteoinductive ability. ${ }^{18-20}$ The recombinant human BMP-2 has been approved by the FDA for various clinical applications, ${ }^{21,22}$ and absorbable gelatin sponge (AGS), used to deliver BMP-2, is the most commercially successful synthetic 
bone graft substitute, due to its demonstrated potency for bone regeneration. ${ }^{19,23}$ However, the clinical application of AGS/ rhBMP-2 is still limited, due to controversy surrounding BMP dosing, complications and cost. ${ }^{21,23}$ Therefore, researchers have turned to gene therapy as an alternative approach to delivering therapeutic proteins. Genetic engineering approaches generally consist of two main types: in vitro and in vivo gene delivery. ${ }^{24}$ In the former, cultured cells are transfected with gene constructs in vitro before they are transplanted into the tissue defect. In the latter, gene constructs are entrapped within a matrix or scaffold without cell pre-transfection. ${ }^{24}$ In our previously study, we prepared BMP-2 gene-activated BMSCs cell sheet in vitro, which were then transplanted to bone defects and found to have a significant effect on promoting bone regeneration in vivo. ${ }^{25}$ However, this strategy takes a long time for cell isolation and culture, and the process of cell sheet preparation is also complicated. Biodegradable scaffolds containing the therapeutic gene, a direct gene transfer strategy and so-called "geneactivated matrix" or GAM have been successfully used in the field of bone tissue engineering. ${ }^{24,26,27}$

Selecting suitable gene vectors is a key challenge in gene therapy. Typically, $25 \mathrm{kDa}$ branched PEI has been regarded as one of the most potent polymer-based gene carriers, being a gold standard due to its unique transfection efficiencies in vitro and in vivo. However, PEI exhibits high cytotoxicity, so many efforts have been made to modify PEI in order to decrease its cytotoxicity whilst retaining the high transfection efficiency. ${ }^{28}$ In our previous study, we prepared polyethyleniminealginate (PEI-al) nanocomposites as a BMP-2 gene vector to transfect bone marrow mesenchymal stem cells (BMSCs), which indicated a significantly enhanced osteogenic differentiation potential of BMSCs. ${ }^{25}$ The results demonstrated that the synthesized PEI-al nanocomposites could efficiently mediate the introduction of the target gene into the BMSCs. Therefore, in the present study, we fabricated a PEI-al/pBMP-2 complexesloaded gelatin sponge and investigated its effect on promoting alveolar bone regeneration, which may be an easy and convenient approach to maintaining the volume and quality of alveolar bone following tooth extraction and alveolar bone surgery.

\section{Material and methods}

\subsection{Preparation and characterization of materials}

2.1.1. Preparation of PEI-al nanocomposites and their DNA complexes. The PEI-al nanocomposites were prepared by electrostatic interactions between PEI (branched PEI $25 \mathrm{kDa}$; Merck KGaA, Darmstadt, Germany) and alginic acid. The preparation procedure and characterization of PEI-al nanocomposites and their DNA complexes were performed as previously reported. ${ }^{27}$ Briefly, alginic acid was first obtained by purification of commercially available sodium alginate (Merck KGaA). Next, $0.9 \mathrm{mg}$ alginic acid was dissolved in $90 \mathrm{ml}$ deionized water and heated to $90{ }^{\circ} \mathrm{C}$ for solubilization, and the alginic acid solution was added dropwise into a preheated $\left(90^{\circ} \mathrm{C}\right)$ solution of PEI (5 $\mathrm{mg}$ dissolved in $500 \mathrm{ml}$ deionized water) under constant stirring at $90{ }^{\circ} \mathrm{C}$ for $4 \mathrm{~h}$. The solution of PEI-al nanocomposites was then concentrated to $20 \mathrm{ml}$ by a rotary evaporator (SENCO,
Shanghai, China) and filtered through a $0.22 \mu \mathrm{m}$ sterile membrane filter for sterilization. PEI-al/DNA complexes were prepared by mixing PEI-al nanocomposites and pDNA (including enhanced green fluorescent protein cDNA plasmid and human $B M P-2$ cDNA plasmid) at different weight ratios (w/ w) and incubating them for $30 \mathrm{~min}$ at room temperature.

2.1.2. Preparation of gelatin sponge loaded with PEI-al/ pBMP-2 complexes. The solution of PEI-al/pBMP-2 complexes was added onto the gelatin sponge and lyophilized after the complexes were absorbed into the gelatin sponge.

2.1.3. Characterization of PEI-al/pBMP-2 complexes and BMP-2 gene-activated AGS. The thermal properties of PEI-al nanocomposites, PEI and alginate were characterized using a thermogravimetric analysis and differential scanning calorimetry (TGA-DSC) instrument (METTLER TGA/DSC 3, Mettler Toledo, Switzerland). Measurements were performed under a nitrogen atmosphere and heated up to $500{ }^{\circ} \mathrm{C}$ at a heating rate of $10{ }^{\circ} \mathrm{C} \mathrm{min}^{-1}$.

The effect of PEI-al nanocomposites on condensation of DNA was performed using PEI-al/pBMP-2 complexes at different w/w ratios of $0.3: 1,0.5: 1,1: 1,1.5: 1,2: 1,5: 1$ and $10: 1$. Each sample was $9 \mu \mathrm{l}$ of PEI-al/pBMP-2 complexes solution plus $1 \mu \mathrm{l}$ of loading buffer $(10 \times)$. Agarose gel retardation assay was investigated by electrophoresis using a $1 \%$ agarose gel at $100 \mathrm{~V}$ for $20 \mathrm{~min}$.

The particle size and zeta potential of the PEI-al nanocomposites, pBMP-2 and PEI-al/pBMP-2 complexes were evaluated by dynamic light scattering (DLS) (Malvern Zetasizer Nano Z; Malvern Instruments, Malvern, UK). PEI-al/pBMP-2 complexes were prepared by mixing PEI-al solution with pBMP-2 at different $w / w$ ratios of $0.3: 1,0.5: 1,1: 1,2: 1,5: 1$ and $10: 1$. The size was assessed by three cycles, and zeta potential was performed by three repeated cycles with 100 runs each.

The morphology of the PEI-al nanocomposites, PEI-al/pBMP2 complexes, gelatin sponge and PEI-al/pBMP-2 complexesloaded gelatin sponge was studied by field emission scanning electron microscopy (SEM; FE-SEM 6700F; JEOL, Ltd., Tokyo, Japan).

\subsection{In vitro studies}

2.2.1. Cell culture. MC3T3-E1 pre-osteoblasts were purchased from the Cell Bank of the Chinese Academy of Sciences (Shanghai, China). They were cultured in $\alpha$-Minimum Essential Medium ( $\alpha$-MEM; Thermo Fisher Scientific, Inc., Waltham, MA, USA), supplemented with $10 \%$ fetal bovine serum (FBS; Thermo Fisher Scientific, Inc.), $100 \mathrm{U} \mathrm{ml}^{-1}$ penicillin and $100 \mathrm{mg} \mathrm{ml} \mathrm{m}^{-1}$ streptomycin (PS; Thermo Fisher Scientific, Inc.). Cells were cultured in a $5 \% \mathrm{CO}_{2}$ atmosphere at $37{ }^{\circ} \mathrm{C}$.

2.2.2. Transfection procedure. MC3T3-E1 cells were seeded in 6-well plates at an initial density of $2 \times 10^{5}$ cells per well in $2 \mathrm{ml}$ DMEM supplemented with 10\% FBS and 1\% PS. Following incubation for $24 \mathrm{~h}$, the media were replaced with serum-free DMEM, previously mixed with PEI-al/pBMP-2 or PEI-al/ enhanced green fluorescent protein cDNA plasmid (pEGFP) 
complexes at different w/w of nanocomposites and plasmid (p) DNA (the amount of plasmid was constant at $2 \mu \mathrm{g}$ ), and the cells were additionally incubated for $4 \mathrm{~h}$. The transfection medium was then exchanged for complete culture medium and incubated for $24 \mathrm{~h}$ at $37^{\circ} \mathrm{C}$.

The transfection efficiency in the cells was demonstrated by flow cytometry. It was performed on PEI-al/pEGFP and PEI/ pEGFP-transfected MC3T3-E1 cells (pEGFP, $2 \mu \mathrm{g}$ per well; PEIal/pEGFP at w/w of $2: 1$ and $3: 1$; PEI/pEGFP, $1: 1$ ). Fortyeight hours following transfection, cells were collected and washed twice with PBS and resuspended in $500 \mu \mathrm{l}$ PBS. The transfection efficiency of the suspended cell samples was then analyzed by flow cytometry (BD Biosciences, San Jose, CA, USA).

2.2.3. Cell viability. An evaluation of cell viability $48 \mathrm{~h}$ post transfection using PEI-al/pBMP-2 complexes was performed using a 3-(4,5-dimethylthiazol-2-yl)-2,5-diphenyltetrazolium bromide (MTT; Amresco, LLC, Solon, OH, USA) cell growth assay. MC3T3-E1 cells $\left(8 \times 10^{3}\right)$ received $200 \mu$ l medium per well, containing $20 \mu \mathrm{l}$ of a $5 \mathrm{mg} \mathrm{ml}^{-1}$ MTT solution, and were incubated for an additional $4 \mathrm{~h}$ at $37{ }^{\circ} \mathrm{C}$. Next, the MTTcontaining medium was replaced with $150 \mu$ l of dimethyl sulfoxide to dissolve the formazan crystals. Absorbance was measured using a microplate reader (Gen5; Biotek Synergy, Winooski, VT, USA) at $490 \mathrm{~nm}$. The percent cell viability of the non-transfected cells taken as $100 \%$.

2.2.4. Studies on transcription and expression levels of BMP-2 gene. BMP-2 gene transcription and expression in MC3T3-E1 cells were studied by qPCR analysis and enzymelinked immunosorbent assay (ELISA) respectively. MC3T3-E1 cells were seeded in 6-well plates at a density of $2 \times 10^{5}$ cells per well. Twenty-four hours later, cells were transfected with PEI-al/pBMP-2 (w/w = $3: 1)$ and PEI-al/pEGFP (w/w = $3: 1)$, as described above. Forty-eight hours later, cells were digested by trypsin and total RNA was isolated from cells using Qiagen RNeasy mini purification kit (Qiagen, Inc., Valencia, CA, USA), according to the manufacturer's instructions. One microgram of RNA was reverse-transcribed using PrimeScript RT Reagent Kit (Takara Biotechnology Co., Ltd., Dalian, China). qPCR was performed by an Mx3005P system using SYBR® Premix Ex Taq ${ }^{\mathrm{TM}}$ (Takara Biotechnology Co., Ltd.), according to the manufacturer's instructions. The conditions of qPCR were as follows: 40 cycles at $94{ }^{\circ} \mathrm{C}$ for $5 \mathrm{~s}, 60{ }^{\circ} \mathrm{C}$ for $34 \mathrm{~s}$. Dissociation stage was added to the end of amplification procedure. There was no nonspecific amplification determined by the dissociation curve. $\beta$-Actin, $5^{\prime}$-CATCCGTAAAGACCTCTATGCCAAC3'/5'-ATGGAGCCACCGATCCACA-3'; human BMP-2, $5^{\prime}$ AACACTGTGCGCAGCTTCC-3' $/ 5^{\prime}$-CCTAAAGCATCTTGCATCTGTTCTC-3', primers were commercially synthesized (Takara Biotechnology Co., Ltd.). The culture medium of cells transfected with PEI-al/pBMP-2 on days 2, 4, 6, 8, 10, 12 and 14 was collected for ELISA analysis. Human BMP-2 concentration was determined by a BMP-2 ELISA kit (R\&D Systems, Inc., Minneapolis, MN, USA), according to the manufacturer's instructions.

2.2.5. Bone-related gene expression studies. MC3T3-E1 cells were seeded in 6-well plates at a density of $2 \times 10^{5}$ cells per well in $2 \mathrm{ml}$ DMEM medium supplemented with 10\% FBS and 1\% PS. Then MC3T3-E1 cells were transfected with PEI-al/pBMP-2 $(\mathrm{w} / \mathrm{w}=3: 1)$, as described above, and cultured in osteogenic medium (50 $\mu \mathrm{g} \mathrm{ml}^{-1}$ ascorbic acid, $10 \mathrm{mM} \beta$-glycerophosphate and $10 \mathrm{nM}$ dexamethasone) for 1,3 and 7 days. The cells transfected with PEI-al/pEGFP were used as the control. At each time point, cells were digested by trypsin and total RNA was isolated from cells using Qiagen RNeasy mini purification kit (Qiagen, Inc.), according to the manufacturer's instructions. One microgram of RNA was reverse-transcribed using PrimeScript RT Reagent Kit (Takara Biotechnology Co., Ltd.). qPCR was performed by an Mx3005P system using SYBR® Premix Ex Taq ${ }^{\mathrm{TM}}$ (Takara Biotechnology Co., Ltd.), according to the manufacturer's instructions. The conditions of qPCR were as follows: 40 cycles at $94{ }^{\circ} \mathrm{C}$ for $5 \mathrm{~s}, 60{ }^{\circ} \mathrm{C}$ for $34 \mathrm{~s}$. Dissociation stage was added to the end of amplification procedure. There was no nonspecific amplification determined by the dissociation curve. $\beta$-Actin was used as the internal control. The primer sequences used for this analysis were as follows: $\beta$-actin, $5^{\prime}$-CATCCGTAAAGACCTCTATGCCAAC- $3^{\prime} / 5^{\prime}$-ATGGAGCCACCGATCCACA-3'; type I collagen (COLL I), 5'-GACATGTTCAGCTTTGTGGACCTC-3'/5'GGGACCCTTAGGCCATTGTGTA-3'; Runt-related transcription factor 2 (RUNX2), 5'-GCACAAACATGGCCAGATTCA-3'/5'-AAGCCATGGTGCCCGTTAG-3'; Osterix (SP7), 5'-AAGTTATGATGACGGGTCAGGTACA-3 ${ }^{\prime} / 5^{\prime}$-AGAAATCTACGAGCAAGGTCTCCAC3'; alkaline phosphatase (ALP), 5'-CTCAACACCAATGTAGCCAAGAATG-3'/5'-GGCAGCGGTTACTGTGGAGA- $3^{\prime}$. Primers were commercially synthesized (Takara Biotechnology Co., Ltd.).

2.2.6. Alkaline phosphatase (ALP) activity and mineralization assay. MC3T3-E1 cells were seeded at a density of $8 \times 10^{3}$ cells per well in 96-well plates or $2 \times 10^{5}$ cells per well in 6 -well plates, and cultured with DMEM medium supplemented with $10 \%$ FBS and 1\% PS. After $24 \mathrm{~h}$, MC3T3-E1 cells were transfected with PEI-al/pEGFP and PEI-al/pBMP-2, as described above, and cultured in osteogenic medium. The ALP activity was analyzed with ALP substrate (Merck KGaA) and read at $520 \mathrm{~nm}$ using a microplate reader (Gen5; Biotek Synergy) on 7 and 14 days following transfection. Alizarin red stain was performed on day 14 following transfection. Cells were fixed with 95\% ethanol and stained with $2 \%$ alizarin red (Merck KGaA).

\subsection{In vivo animal experiments}

2.3.1. Preparation of implants. PEI-al/pBMP-2 (w/w of $2: 1$, $10 \mu \mathrm{g}$ of pBMP-2) and PEI-al $(20 \mu \mathrm{g})$ solutions were absorbed into a cuboid gelatin sponge $15 \mathrm{~mm}$ in length and $2 \mathrm{~mm}$ in width and height, and then dried using a vacuum pump.

2.3.2. Animals. The animal experiments used in this study were approved by the Ethics Committee of the Second Affiliated Hospital of Harbin Medical University (Harbin, China; approval no. 2015-yan-018), and were carried out under the control of the University's Guidelines for Animal Experimentation. Twentyseven male Wistar rats (average weight, $220 \mathrm{~g}$ ) were used. The animals were maintained at a temperature of $22{ }^{\circ} \mathrm{C}$ on a $12 / 12$ hour light/dark cycle and fed with a standard laboratory diet and water.

2.3.3. Surgical procedure. The in vivo study was designed following the Animal Research: Reporting In Vivo Experiments guidelines. The animal model was established according to the 
method of alveolar bone regeneration by Elsubeihi and Heersche. ${ }^{29}$ Specifically, the animals were anesthetized with intraperitoneal injection of ketamine $\left(60 \mathrm{mg} \mathrm{kg}^{-1}\right)$ and xylazine $\left(8 \mathrm{mg} \mathrm{kg}^{-1}\right)$. The crown of the left mandibular incisor was cut down at the gingival level using a high-speed turbine drill; this procedure was repeated every 2 days until this mandibular incisor was fully extracted on day 12 . The animals were randomly divided into three groups: control group, unfilled sockets $(n=9)$; AGS group, sockets filled with PEI-al solutionloaded gelatin sponges $(n=9)$; AGS/BMP group, sockets filled with PEI-al/pBMP-2 solution-loaded gelatin sponge $(n=9)$. The sockets were then covered with periodontal dressing.

One week after surgery, three rats were sacrificed in each group. A $0.25 \mathrm{~cm}^{2}$ square bone tissue, under the first molar region of the mandible encompassing the incisor tooth socket, was harvested for western blot to determine the expression of BMP-2 protein in alveolar socket (Fig. 5A). After the bone tissues were crushed in liquid nitrogen, the protein was extracted using the tissue protein extraction kit (Solarbio Science \& Technology Co., Ltd, Beijing, China). The protein samples (50 $\mu \mathrm{g})$ was separated by $12 \%$ of SDS-PAGE gel (Beyotime Biotechnology, Jiangsu, China) and transferred onto nitrocellulose blotting membrane (Beyotime Biotechnology, China). After blocking with $5 \%$ non-fat dry milk at room temperature for $1 \mathrm{~h}$, the membrane was incubated overnight at $4{ }^{\circ} \mathrm{C}$ with the primary antibody of human BMP-2 (R\&D Systems, Inc.) $(1$ : 1000) and $\beta$-actin (Cell Signaling Technology, Inc., Danvers, MA, USA) (1:1000), followed by the corresponding secondary antibody. The protein signals were visualized by Chemiluminescent Imaging System (Tanon Science \& Technology Co., Ltd., Shanghai, China).

At 4 and 8 weeks, the rats were sacrificed by heart perfusion fixation with $4 \%$ paraformaldehyde solution. The mandibles were removed and fixed in $4 \%$ paraformaldehyde solution. The mandible samples were examined by soft X-ray (Shenzhen Macro electronic Co., Ltd., Shenzhen, China), in order to calculate the relative height of the residual alveolar ridge, and LEXXOS Digital 2D Densitometer (Diagnostic Medical Systems, Montpellier, France) for BMD. The relative height of the residual alveolar ridge was evaluated by the ratio of the height of the alveolar ridge on the left and right sides of the mandible. The height of the alveolar ridge was the distance from the apex of the alveolar ridge of the mandibular incisor to the apex of the mesial alveolar bone of the first molar (Fig. 5C). The data were measured by ImagePro Plus 6.0 software (IPP). A $0.33 \mathrm{~cm}^{2}$ round area, under the first molar region of the mandible encompassing the incisor tooth socket, was selected for BMD measurement (Fig. 5E). The anterior border of the area was determined to be a flat plane, approximately $100^{\circ}$ across the occlusal plane of the molar teeth, as shown in Fig. 5E. Next, the tooth extraction side of the mandible was decalcified, embedded, sliced and stained with hematoxylin and eosin (HE) staining.

\subsection{Statistical analysis}

Results are expressed as the mean \pm standard deviation (SD). One-way analysis of variance followed by Tukey's post hoc test was used to determine the statistical significance of differences between groups. $P<0.05$ was considered to indicate a statistically significant difference. The Statistical Package for Social Science, version 21.0 (SPSS Inc., Chicago, IL, USA) was used for all analysis.

\section{Results}

3.1. Characterization of PEI-al/pBMP-2 complexes and BMP2 gene-activated AGS

PEI-al nanocomposites are formed by ionic interaction between the amino band (- $\mathrm{NH}_{2}$ and $\left.-\mathrm{NH}-\right)$ of PEI and the carbonyl band $(-\mathrm{COOH})$ of alginate, which has been proved by Fourier transform infrared in our previous study. ${ }^{25}$ The thermal properties of PEI-al nanocomposites, PEI and alginate were also investigated by TGA-DSC. As indicated in Fig. S1 $\dagger$ that PEI-al nanocomposites started to be decomposed at about $100{ }^{\circ} \mathrm{C}$ and $340{ }^{\circ} \mathrm{C}$, while alginate and PEI started to decompose at about $180{ }^{\circ} \mathrm{C}$ and $300{ }^{\circ} \mathrm{C}$, respectively. The formation of the PEI-al/ pBMP-2 complexes at different $\mathrm{w} / \mathrm{w}$ ratios were confirmed using agarose gel retardation assay and their particle size and zeta potential were evaluated. Results of electrophoresis shows that the migration of BMP-2 plasmid was almost completely retarded when the $\mathrm{w} / \mathrm{w}$ ratio was reached $1: 1$ (Fig. 1A). It suggests the formation of neutral or positively charged complexes between PEI-al and BMP-2 plasmid when the w/w ratio reached $1: 1$. This is consistent with the results of DLS (Fig. 1B), zeta potential of PEI-al/pBMP-2 complexes was negative when the $\mathrm{w} / \mathrm{w}$ ratios were $0.3: 1$ and $0.5: 1$, where the complexes could not form completely. With the increasing of the $\mathrm{w} / \mathrm{w}$ ratio, the zeta potential of the complexes rapidly increased to positive values. The particle size of PEI-al/pBMP-2 complexes also decreased rapidly when the $\mathrm{w} / \mathrm{w}$ ratio was from $0.3: 1$ to $1: 1$, and then decreased gradually with the increase of the $\mathrm{w} / \mathrm{w}$ ratio.

SEM images showed that PEI-al nanocomposites were dispersed nanoparticles, whereas their DNA complexes were aggregated (Fig. 2A and B). The gelatin sponge had a 3D interconnected porous structure with a pore size of $100-200 \mu \mathrm{m}$ (Fig. 2C). PEI-al/pBMP-2 complexes were localized in the gelatin sponge (Fig. 2D).

\subsection{Results of in vitro studies}

3.2.1. Transfection efficiency of PEI-al nanocomposites in MC3T3-E1 cells. In the present study the transfection efficiency of the PEI-al nanocomposites was examined on an MC3T3-E1 cell line (Fig. 3A) using a plasmid DNA-containing reporter gene encoding EGFP. Flow cytometry showed that the transfection efficiency of PEI-al/pEGFP at w/w of $2: 1$ and $3: 1$ was 19.75\% (Fig. 3C) and 19.13\% (Fig. 3D), respectively, in MC3T3E1 cells, whereas that of PEI/pEGFP (w/w = $1: 1)$ was $5.48 \%$ (Fig. 3B).

3.2.2. Cytotoxicity of PEI-al/pBMP in MC3T3-E1 cells. The post-transfection cytotoxicity against cells has always been a concern with any vector/pDNA system. To assess the cytotoxicity of PEI-al nanocomposites, we evaluated relative cell 

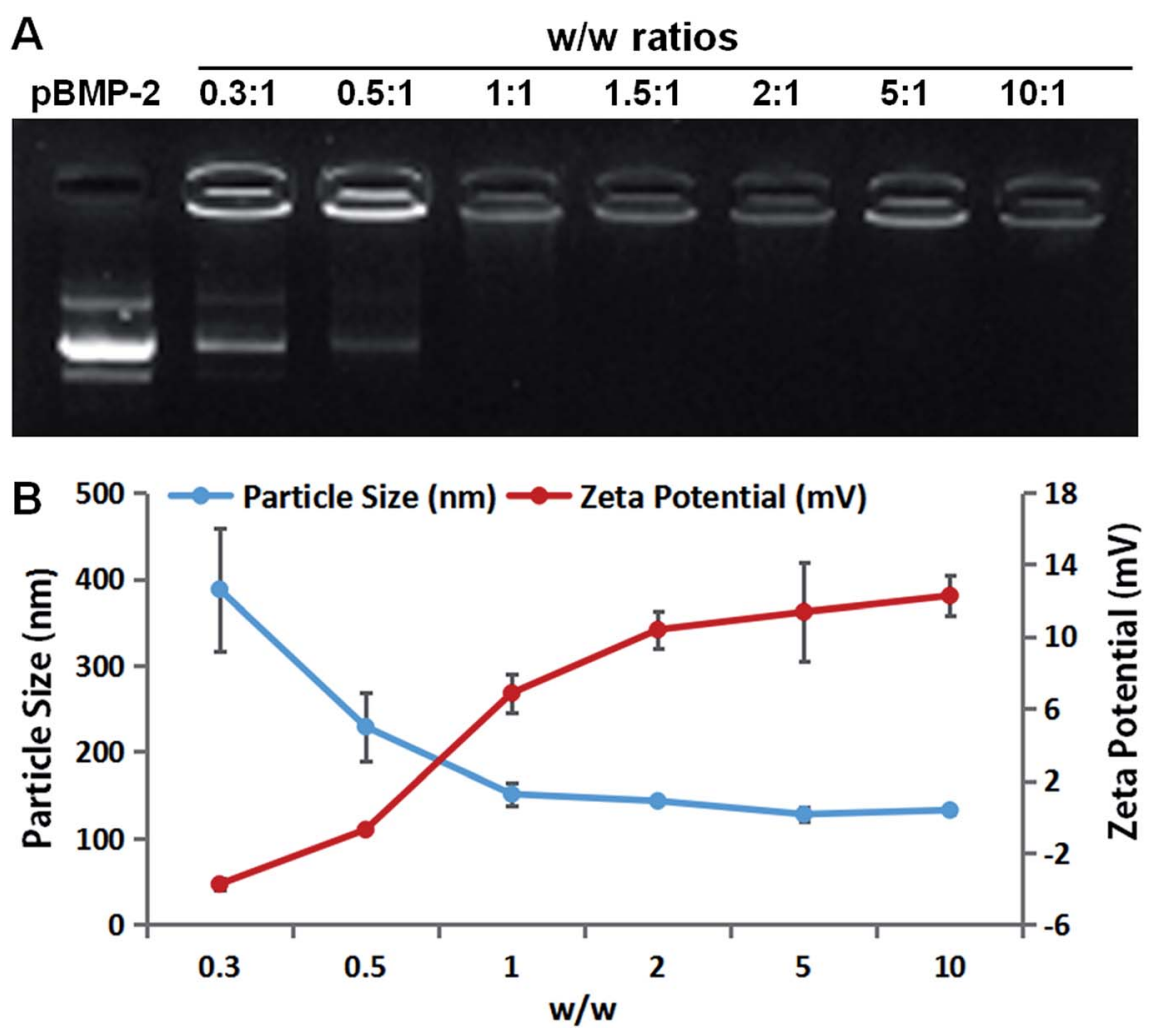

Fig. 1 Characterization of the PEI-al nanocomposites and PEI-al/pBMP-2 complexes. (A) Agarose gel electrophoresis retardation assay of PEIal/pBMP-2 at various $\mathrm{w} / \mathrm{w}$ ratios. (B) Particle size and zeta potential of PEI-al/pBMP-2 at various w/w ratios. Data are reported as mean \pm SD $(n=$ 3).

viability by MTT assay. As shown in Fig. 3E, at w/w of $0.5: 1$, $1: 1,2: 1$ and $3: 1$, PEI-al/pBMP nanocomposites displayed an almost identical viability as non-transfected cells (given as
$100 \%$ ). By contrast, the cytotoxicity of PEI/pBMP-2 was significant, as compared with PEI-al/pBMP nanocomposites and untreated cells at $\mathrm{w} / \mathrm{w}$ of $>2: 1(P<0.01)$.

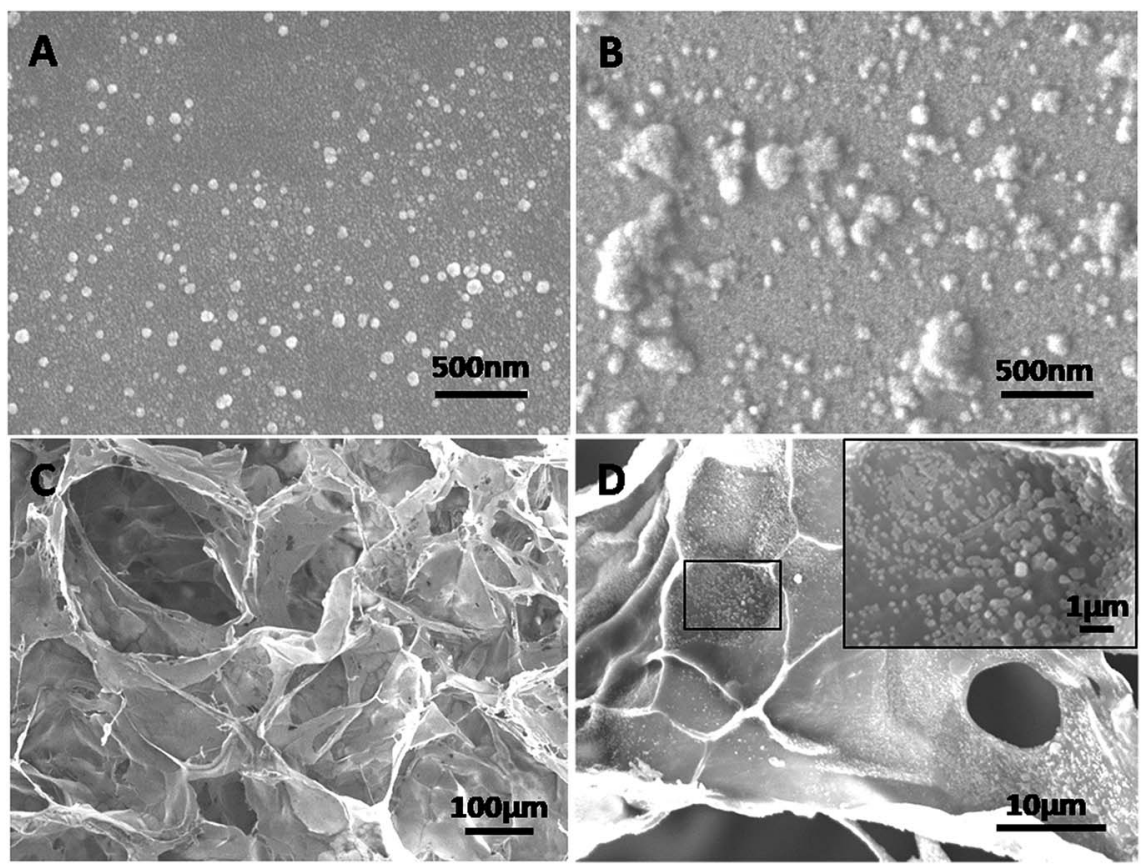

Fig. 2 Morphology of PEI-al/pBMP-2 complexes and BMP-2 gene-activated gelatin sponge. SEM images of (A) PEI-al nanocomposites, (B) PEIal/pBMP-2 complexes, (C) gelatin sponge and (D) gelatin sponge loaded with PEI-al/pBMP-2 complexes. 


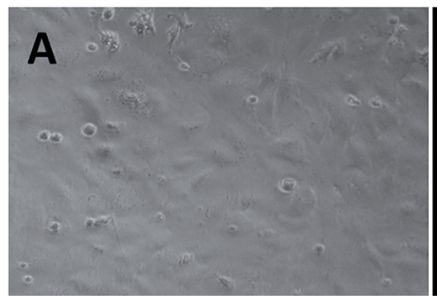

E

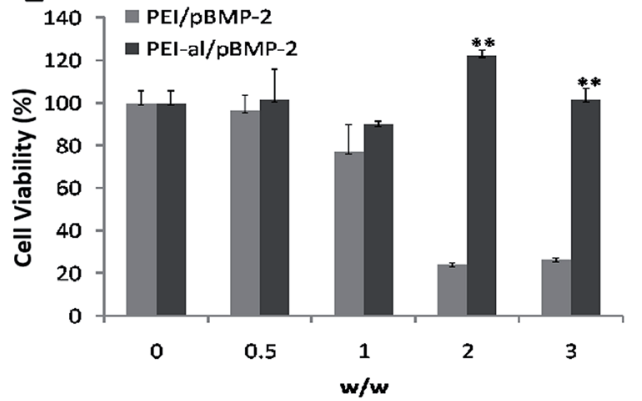

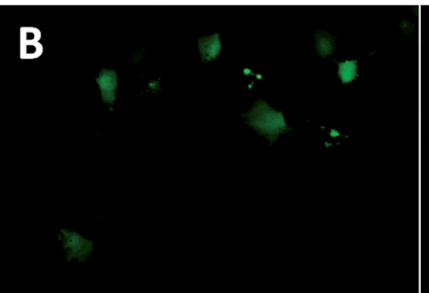

$\mathbf{F}$

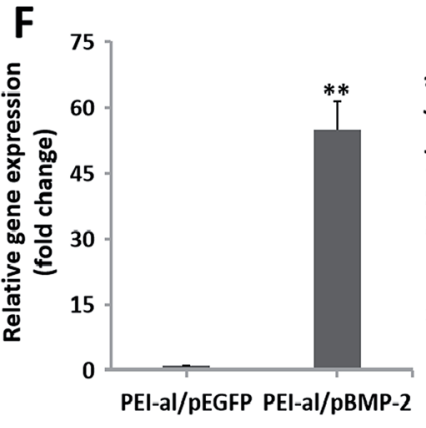

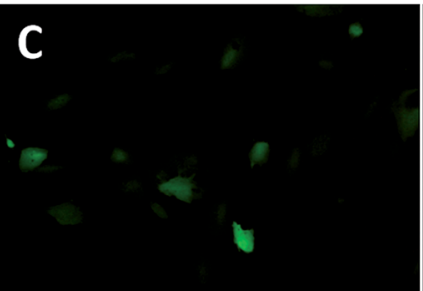
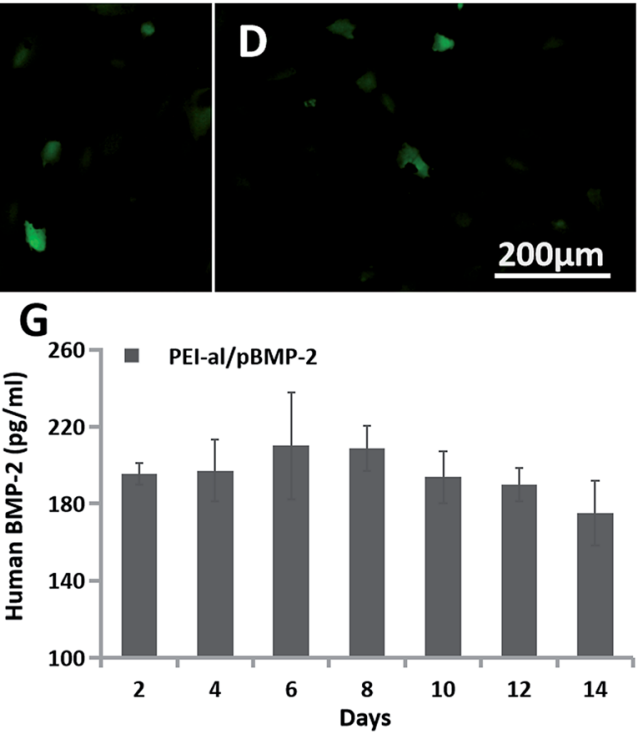

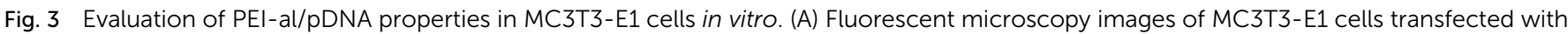

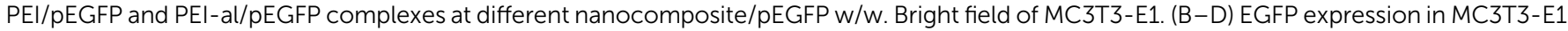

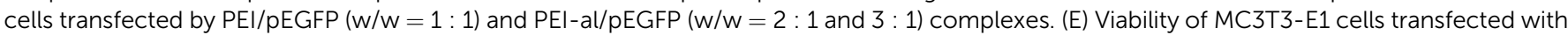

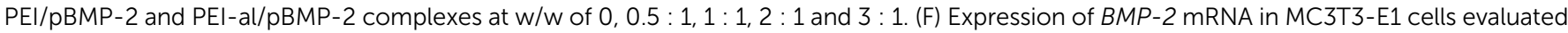

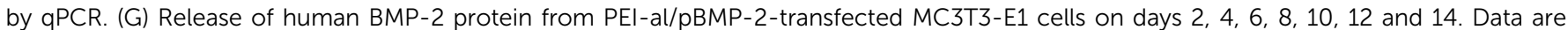
reported as mean $\pm \mathrm{SD}(n=3), * P<0.05$ and $* * P<0.01$.

3.2.3. The transcription and expression levels of human BMP-2 mRNA and protein in transfected MC3T3-E1 cells. Due to the high homology of the $B M P-2$ gene in mice and humans, our primers designed for human $B M P-2$ mRNA can also amplify the $B M P-2$ gene of mice. Result from Fig. $3 \mathrm{~F}$ shows the $B M P-2$ gene expression in the PEI-al/pBMP-2 group was significantly higher than that in the PEI-al/pEGFP group $(P<0.01)$. Fig. 3G shows the concentration of human BMP-2 protein secreted from MC3T3-E1 cells on days 2, 4, 6, 8, 10, 12 and 14 posttransfection with PEI-al/pBMP-2 (w/w = $3: 1)$, which indicated that the secretion of BMP-2 protein could last for a minimum of 14 days.

3.2.4. Effects of PEI-al/pBMP on osteogenic differentiation in MC3T3-E1 cells. The relative expression of osteogenic genes was evaluated by qPCR 1,3 and 7 days following the transfection of MC3T3-E1 cells with PEI-al/pBMP-2 and PEIal/pEGFP. Fig. 4A shows that the gene expression of COLL I $(P<0.01), S P 7(P<0.01)$ and $R U N X 2(P<0.01)$ in the PEI-al/ pBMP-2 group was significantly increased on day 1 , as compared with the PEI-al/pEGFP group $(P<0.01)$, while there was no difference in the expression of $A L P$ between the two groups. On day 3 , the expression of COLL $I(P<0.01)$ and RUNX2 $(P<0.01)$ in the PEI-al/pBMP-2 group was still higher than those in the PEI-al/pEGFP group $(P<0.01)$, but there was no difference in that of $S P 7$ and $A L P$ between the two groups (Fig. 4B). On day 7, the expression of COLLI $(P<0.01), S P 7(P<$ $0.05)$ and $A L P(P<0.01)$ in the $P E I-a l / p B M P-2$ group was higher than that in the PEI-al/pEGFP group $(P<0.05)$. However, the expression of RUNX2 was decreased, and the expressions of the PEI-al/pBMP-2 group and the PEI-al/ pEGFP group were no difference (Fig. 4C).
ALP is also an important indicator of osteoblastic activity. Fig. 4D shows ALP activity in the PEI-al/pBMP-2 group, as compared to the PEI-al/pEGFP group, on days 7 and 14. ALP activity in the PEI-al/pBMP-2 group was significantly higher than that in the PEI-al/pEGFP group on days $7(P<0.01)$ and 14 $(P<0.01)$.

The calcium deposition of MC3T3-E1 cells following transfection with PEI-al/pBMP-2 and PEI-al/pEGFP was evaluated by alizarin red staining. Fig. $4 \mathrm{E}$ and $\mathrm{F}$ shows that the PEI-al/pBMP2 group had more red calcium deposition than the PEI-al/ pEGFP group.

\subsection{Results of in vivo studies}

Wound healing following tooth extraction was uneventful. Western blot analysis shows BMP-2 protein was only expressed in bone tissues of AGS/BMP group, indicating that BMP-2 gene could be transfected into local cells and express target protein in vivo (Fig. 5B). The relative height of the residual alveolar ridge in the AGS/BMP, AGS and control groups was measured by IPP. The results showed that the relative height of the residual alveolar ridge was significantly higher in the AGS/BMP group than that in the AGS and control groups at 4 (AGS/BMP vs. AGS, $P<0.05$; AGS/BMP vs. control, $P<0.01$ ) and 8 weeks (AGS/BMP vs. AGS, $P<0.01$; AGS/BMP vs. control, $P<0.01)$. No differences were observed between the AGS and control groups (Fig. 5D). The result of BMD analysis also demonstrated that the AGS/ BMP group was significantly higher than the AGS and control groups at 4 (AGS/BMP vs. AGS, $P<0.05$; AGS/BMP vs. control, $P<$ 0.05 ) and 8 weeks (AGS/BMP vs. AGS, $P<0.05$; AGS/BMP vs. control, $P<0.01)$. No differences were observed between the AGS and control groups (Fig. 5F). Further histology indicated 

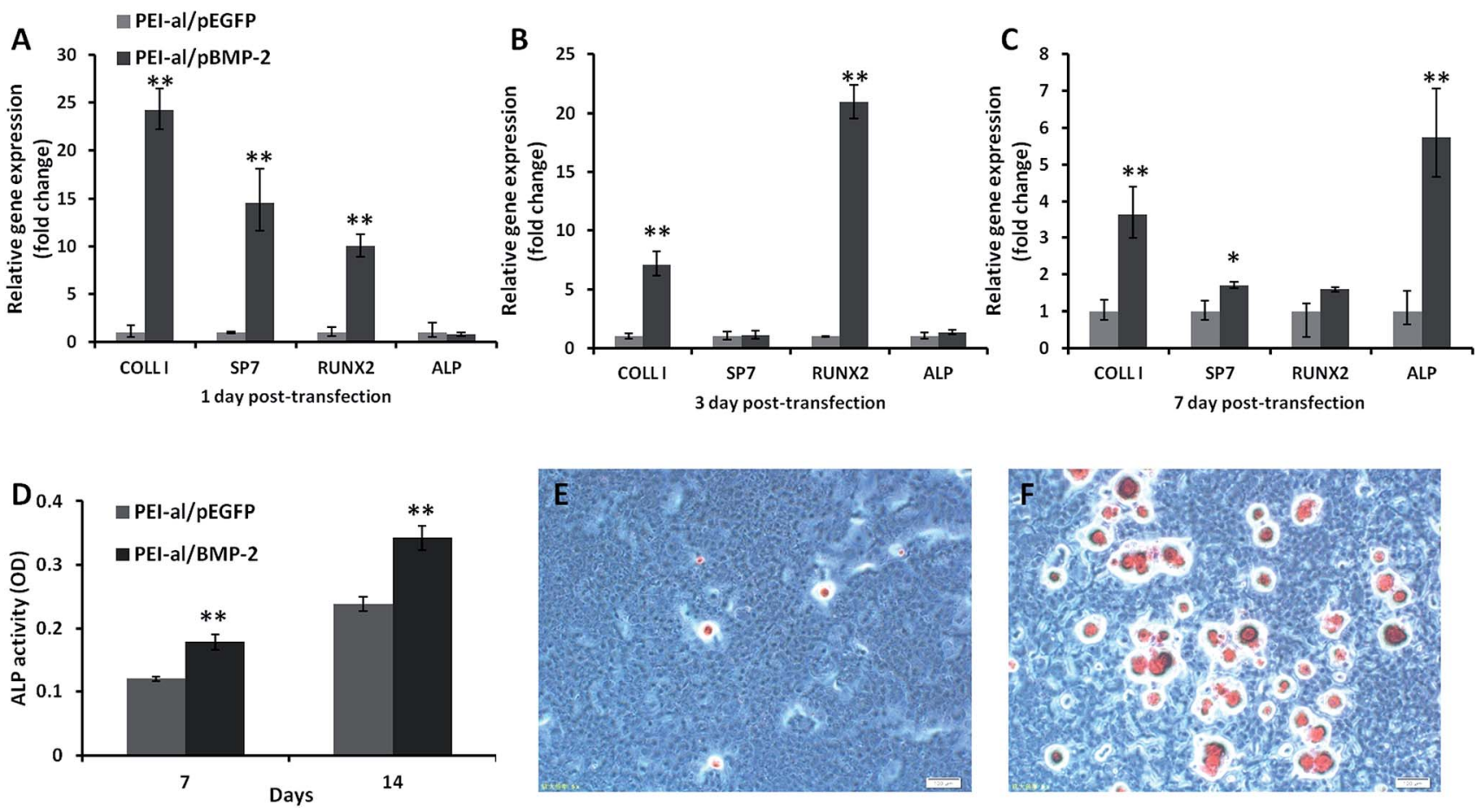

Fig. 4 Effects of PEI-al/pBMP on osteogenic differentiation in MC3T3-E1 cells. Expression of specific osteogenic genes (COLL I, SP7, RUNX2, $A L P$ ) in MC3T3-E1 cells evaluated by qPCR on days (A) 1, (B) 3 and (C) 7 post-transfection with PEI-al/pEGFP and PEI-al/pBMP-2 complexes. (D) ALP activity was analyzed on days 7 and 14. Alizarin red staining was performed on day 14 post-transfection with (E) PEI-al/pEGFP and (F) PEI-al/ pBMP-2 complexes. Data are reported as mean $\pm \mathrm{SD}(n=3), * P<0.05$ and $* * P<0.01$.

that the bone formation in the defect area was significantly increased in the AGS/BMP group, as compared to the AGS and control groups 4 and 8 weeks post-operatively (Fig. 6A-F). At 4 weeks, osteoblasts around the newly formed bone trabeculae were cubic-shaped in the AGS/BMP group, which indicated active osteogenesis (Fig. 6G).

\section{Discussion}

Satisfactory and immediate treatment with alveolar bone defects are essential to maintaining an adequate volume and morphology of the alveolar bone. ${ }^{1}$ Osteogenic growth factors, such as BMPs, transforming growth factor $\beta$, platelet-derived growth factor (PDGF) and vascular endothelial growth factor are often used to mediate bone development and repair. ${ }^{24,25}$ Kumasaka and colleagues ${ }^{30}$ reported that continuous subcutaneous infusion insulin-like growth factor I (320 $\mathrm{mg}$ per day) for 3 weeks by osmotic minipump to inhibit alveolar bone loss following tooth extraction in rats. Because of the biological halflife of growth factors ranges between several minutes and several hours. ${ }^{22,24}$ Therefore, to achieve a therapeutic effect, a large dose or repeat application of proteins is always required, which hampers their clinical use. ${ }^{21,22}$ Liu et al. ${ }^{31}$ used BMP-2 to induce osteogenic differentiation of dental pulp stem cells in vitro, and then combined with the tissue engineering scaffold to repair the alveolar bone defect. Although the method can avoid the side effects caused by the extensive application of the BMP-2 in vivo, it takes a long time to isolate and culture cells, and the process of constructing tissue engineered bone is also relatively complex. In order to avoid these disadvantages, the $B M P-2$ gene was used instead of the BMP-2 protein to induce bone formation in the present study. When the BMP-2 gene is transferred to local cells, the secreted BMP-2 protein can stimulate osteogenesis for a longer time than that seen in protein therapy. ${ }^{32}$ However, the direct injection of BMP-2 gene solution into defects for their regeneration has a poor therapeutic effective. This is due to the fact that the water-soluble factors cannot be maintained at the injection site. Gene therapy is often combined with bone tissue engineering scaffolds to fabricate GAM for the enhancement of the bone repairing ability. ${ }^{25,26}$ Due to flexibility in shape, biocompatibility, biodegradability and affinity to proteins and growth factors, gelatin sponges may be excellent candidates for bone graft scaffolds in low-load areas or as drug delivery materials. When a gelatin sponge is combined with the $B M P-2$ gene, it forms a biologically matrix. ${ }^{13}$ Following its transplantation to the defect, the matrix not only holds the $B M P$-2 gene but also maintains its bioactivity, until endogenous cells migrate through the matrix, are transfected and secrete BMP-2 protein. Furthermore, in this study, BMP-2 gene was used as a model molecule, which could be replaced by other bone-induced growth factors or combined with other factors to play a synergistic role. And we can easily prepare a variety of osteoinductive gene-activated matrices. Moreover, the geneactivated gelatin sponge developed in the present study was easy to prepare and store, and can be directly used to fill bone defects immediately following tooth extraction. Therefore, this 

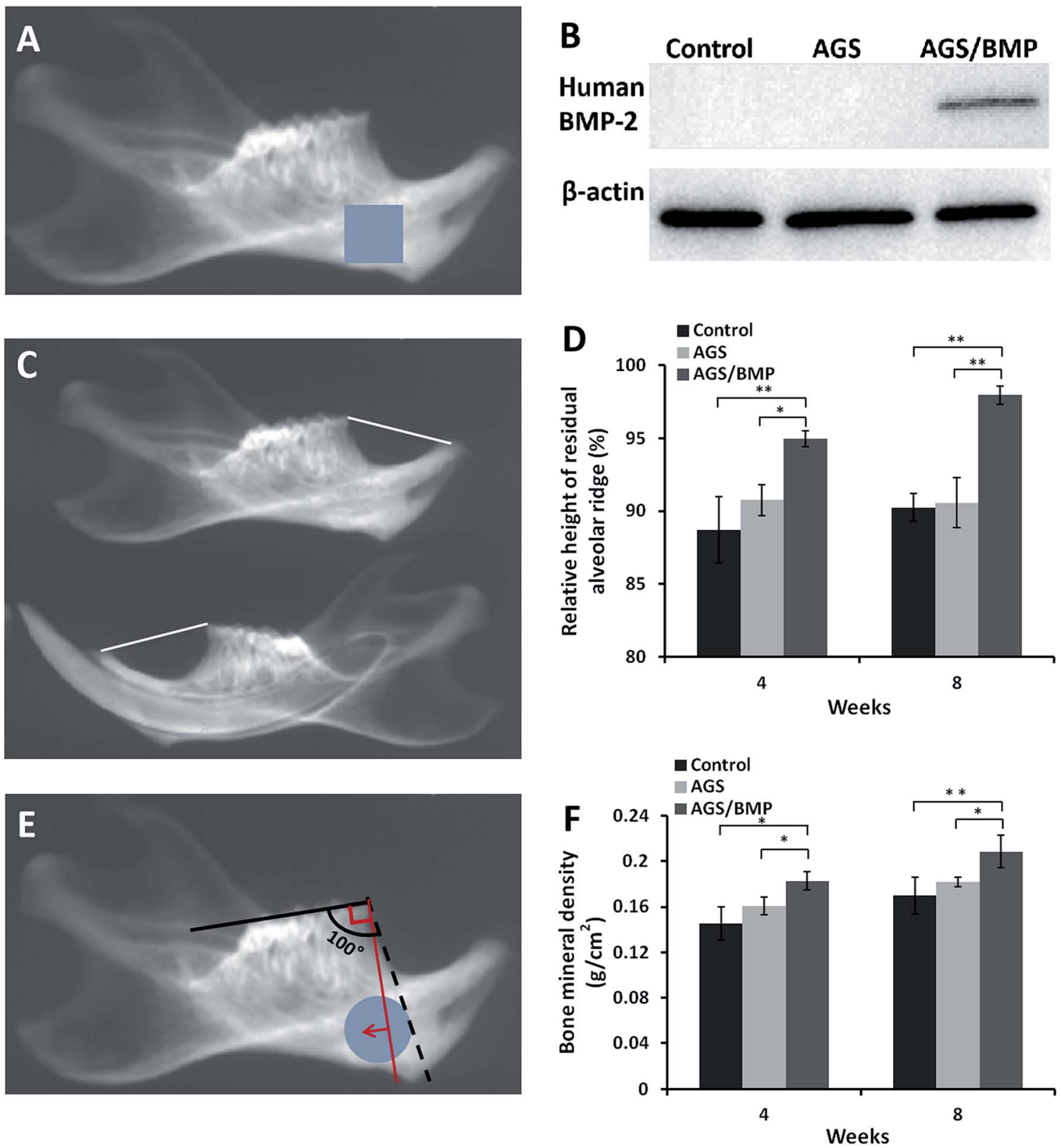

Fig. 5 Data from in vivo animal experiments. (A) Photo of soft X-ray shows the tooth extraction side of the mandible. The square blue area was harvested for western blot. (B) Expression of human BMP-2 protein in vivo. (C) Photo of soft X-ray shows the left and right sides of the mandible. The white line is the height of the mandibular residual ridge. (D) Measurement of the relative height of the residual alveolar ridge. (E) Photo of soft $X$-ray shows the tooth extraction side of the mandible. The round blue area was selected to measure BMD. The red line shows the direction of the histological section. (F) BMD results. Data are reported as mean $\pm \mathrm{SD}(n=3), * P<0.05$ and $* * P<0.01$.

therapeutic strategy provides a simple and convenient procedure for the regeneration of the alveolar bone.

The majority of gene therapy treatments for bone repair have used adenoviral vectors. Dunn $e t$ al. $^{33}$ used a collagen matrix to deliver a recombinant adenoviral vector encoding BMP-7 to alveolar bone defects. Although adenovirus is an efficient means of gene transfer, it also elicits a significant immune response mediated by cytotoxic $\mathrm{T}$ cells. ${ }^{34}$ Therefore, gene therapy based on non-viral gene vectors is preferred by more and more researchers due to its reported low immunogenicity, transient expression without genetic recombination and production of large protein quantities at a low cost. ${ }^{26}$ In the present study, the PEI-al nanocomposites were used as to nonviral gene vectors to deliver $B M P-2$ plasmid. PEI exhibits high cytotoxicity, so many efforts have been made to modify PEI in order to decrease its cytotoxicity whilst retaining the high transfection efficiency. It is generally thought that polycation cytotoxicity is caused by polymer aggregation on the cell surface due to the strong electrostatic interaction with plasma membrane, which impairs the cell membrane functions. ${ }^{27}$ For this reason, in this study, PEI-al nanocomposites were synthesized by electrostatic interaction between cationic polymer PEI 

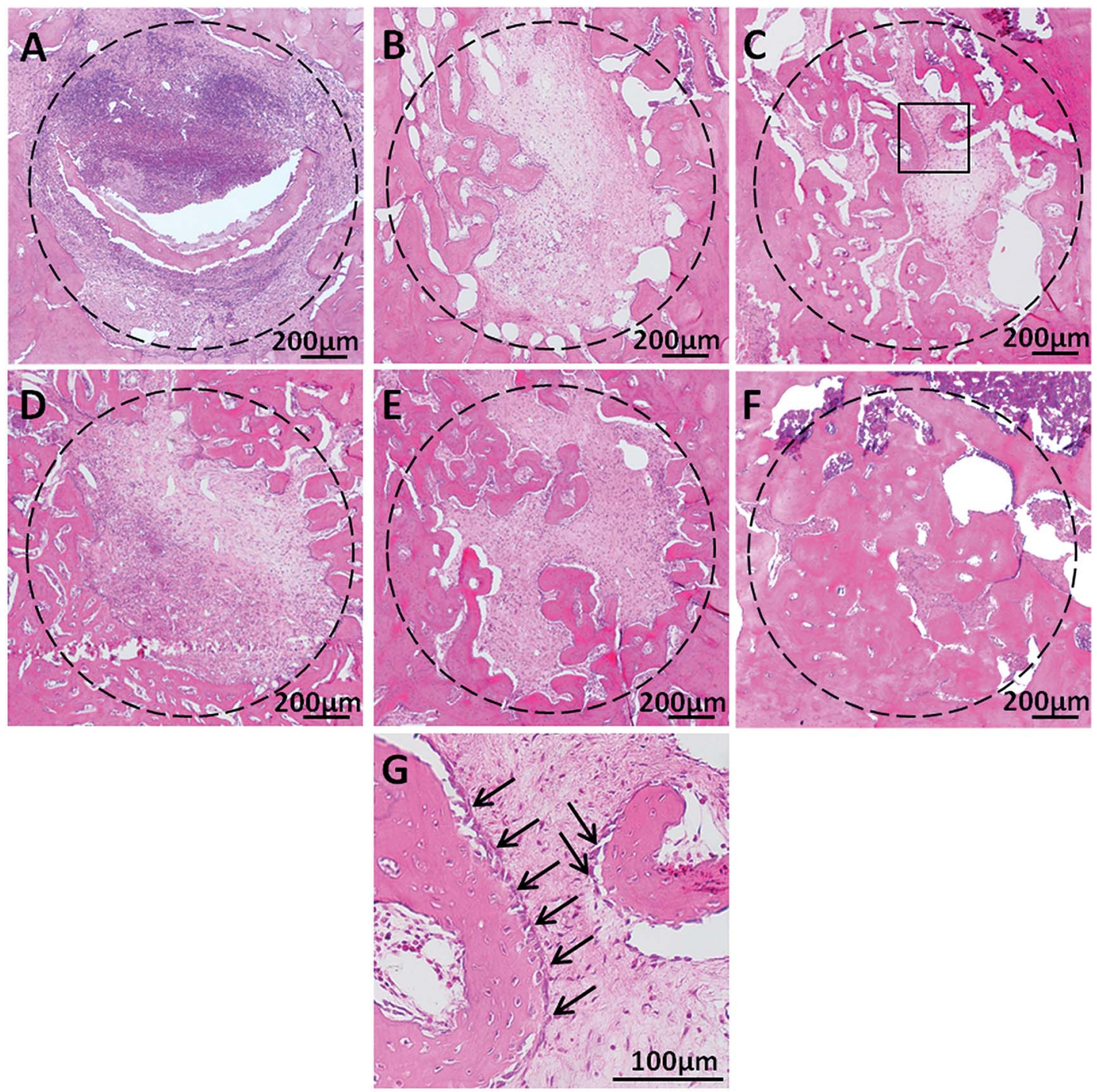

Fig. 6 Hematoxylin and eosin staining of the defect area 4 and 8 weeks post-operatively. (A) Control, (B) AGS and (C) AGS/BMP groups at 4 weeks. (D) Control, (E) AGS and (F) AGS/BMP groups at 8 weeks. (G) Enlarged image of the newly formed bone in the AGS/BMP group at 4 weeks. Black arrows indicate osteoblasts. AGS, absorbable gelatin sponge.

and anionic polymer alginate, and blending polyanionic alginate with PEI successfully shielded the excess positive charge on PEI and then reduced its toxicity. In vitro studies have proven the PEI-al nanocomposites had relatively higher transduction efficiency and lower cytotoxicity than PEI in MC3T3-E1 cells. In animal experiments, we also collected liver, spleen and kidney tissues of rats to evaluate the in vivo toxicity of PEI-al/pBMP-2 complexes. The results showed that there was no significant difference in histopathological changes of the tissue samples among the control group, AGS group and AGS/BMP group (Fig. S2 $\dagger$ ), which further indicated that PEI-al/pBMP-2 complexes had good biosafety in vivo.

When the BMP-2 gene expression has been emerging, the protein secretion begins and then osteogenesis is stimulated by autocrine and paracrine pathways. ${ }^{35}$ Further evaluation showed that $B M P-2$ gene transduction can promote the osteogenic differentiation of preosteoblasts, as demonstrated by the increased expression of specific bone markers RUNX2, SP7, COLL I and ALP, as well as ALP activity and calcium nodule formation at the different time points. ${ }^{36}$ RUNX2 is known as the main regulator of osteoblast differentiation, and its expression is induced by BMP-2. ${ }^{35}$ During the process of osteoblast differentiation, RUNX2 is further involved in the expression of $S P 7$, COLL I, osteopontin, bone sialoprotein and osteocalcin. ${ }^{36,37}$ However, at the late stages of osteoblast differentiation RUNX2 inhibits the differentiation of the immature osteoblasts into mature osteoblasts and osteocytes. ${ }^{37}$ The results of the present study also indicated that the expression of osteoblast marker genes RUNX2, SP7 and COLL I was significantly increased at the early stage of osteoblast differentiation, while that of RUNX2 was decreased at the later stage (Fig. 4A-C). The expression of the $A L P$ gene and ALP activity were then increased (Fig. 4D), which may have promoted the mid- and late-stage osteoblast maturation and calcium deposition. ${ }^{38}$ 
GAM is one of the in vivo gene delivery systems and has been successfully used for tissue regeneration. When GAM is implanted into the tissue defect, it forms a biologically active matrix. $^{24,25}$ The GAM pore architecture provides a 3D matrix to promote cell ingrowth and delivers the entrapped plasmid DNA to stimulate local protein production. ${ }^{39}$ The matrix used in our study was a gelatin sponge, which is commonly applied in the clinic for hemostasis immediately following tooth extraction. Its pore size is $100-200 \mu \mathrm{m}$, which is suitable for cell infiltration and ingrowth of host bone tissue. ${ }^{\mathbf{4 0 , 4 1}}$ In an early study, Stanton et al. reported that gelatin sponge played the role of a scaffold in supporting chondrocyte and osteoblast cells in vitro. ${ }^{\mathbf{1 4}}$ Other studies have demonstrated that the ability of the gelatin scaffold to induce osteogenesis could be significantly improved by incorporating it with BMPs in vitro and in vivo. ${ }^{13}$ In the present study, a gelatin sponge loaded with the $B M P-2$ gene was implanted into the incisor socket of the rats immediately following tooth extraction. It soaked blood and was populated by infiltrating cells of the host. With the secretion of the BMP-2 protein from transfected local cells, a series of cellular events were activated, including recruiting undifferentiated mesenchymal and other target cells into the gelatin sponge and inducing their differentiation into osteoblasts. ${ }^{39,40}$ In cases of bone repair, there are two types of osteogenesis, which are very similar to intramembranous and endochondral ossification during embryogenesis. In the process of intramembranous ossification, osteoblasts are directly differentiated from mesenchymal cells, while in the process of endochondral ossification, mesenchymal cells first differentiate into chondrocytes and form a cartilage template. When chondrocytes in the template mature, the surrounding mesenchymal cells immediately differentiate into osteoblasts, suggesting that osteoblasts and chondrocytes derive from a common progenitor cell. ${ }^{42}$ Cartilage phenotype expression has been observed during intramembranous bone formation in the sockets of rats, and endochondral bone formation by rhBMP-2 has been observed in rat mandibular defects. ${ }^{43}$ However, several studies have found that BMPs stimulate only intramembranous bone formation during bone healing in animal models of calvarial and periodontal defects. ${ }^{44}$ One reason may be that periodontal ligament fibroblasts and endosteal fibroblastic cells in the rat mandible are osteoprogenitors committed to directly inducing bone regeneration; however, this remains to be elucidated. ${ }^{45}$ The concentration of rhBMP-2 may be another reason for the different osteogenesis of mandibular defects in rats. When a high concentration of BMP-2 was used, bone healing was primarily based on intramembranous ossification, rather than endochondral bone formation. ${ }^{\mathbf{4 6}}$ Similarly, endochondral ossification was not observed in the present study. HE staining observed active osteoblasts surrounding the new bone, indicating that intramembranous ossification may have occurred.

\section{Conclusion}

The combined use of PEI-al/pBMP-2 complexes and gelatin sponge is an effective method for bone regeneration, which may provide an easy and convenient approach to maintaining the volume and quality of alveolar bone following tooth extraction and alveolar bone surgery. Further clinical studies comparing this material with other known techniques to confirm the effectiveness of the material used in clinical treatment.

\section{Conflicts of interest}

The author reports no conflicts of interest in this work.

\section{Acknowledgements}

This work was supported by the National Natural Science Foundation of China (Grant No. 81500816, 81570951), the Harbin Applied Technology Research and Development Project (Grant No. 2017RAQXJ237), the Innovation Science Foundation of Harbin Medical University (Grant No. 2016LCZX10), Health and Family Planning Commission of Heilongjiang Provincial (Grant No. 2018031), The Fundamental Research Funds for the Provincial Universities.

\section{References}

1 D. Clark, Y. Rajendran, S. Paydar, S. Ho, D. Cox, M. Ryder, J. Dollard and R. Kao, J. Periodontol., 2018, 89, 379-387.

2 J. Hao, J. Chou, S. Kuroda, M. Otsuka, S. Kasugai and N. P. Lang, Clin. Oral Implant. Res., 2018, 29, 227-234.

3 F. Wang, L. Du and S. Ge, Sci. Rep., 2016, 6, 30403.

4 F. Wang, Y. Zhou, J. Zhou, M. Xu, W. Zheng, W. Huang, W. Zhou, Y. Shen, K. Zhao, Y. Wu and D. Zou, J. Dent. Res., 2018, 97, 1229-1235.

5 J. K. Petersen, J. Krogsgaard, K. M. Nielsen and E. B. Nørgaard, Int. J. Oral Surg., 1984, 13, 406-410.

6 S. Sae-Jung and P. Apiwatanakul, Asian Spine J., 2018, 12, 195-201.

7 D. S. Sohn, J. W. Moon, K. N. Moon, S. C. Cho and P. S. Kang, J. Oral Maxillofac. Surg., 2010, 68, 1327-1333.

8 L. Cui, P. Chen, Z. Tan, W. Li and Z. Dong, Prostate, 2012, 72, 1669-1677.

9 C. Jinno, N. Morimoto, R. Ito, M. Sakamoto, S. Ogino, T. Taira and S. Suzuki, BioMed Res. Int., 2016, 2016, 4567146.

10 S. Miyayama, K. Yamakado, H. Anai, D. Abo, T. Minami, H. Takaki, T. Kodama, T. Yamanaka, H. Nishiofuku, K. Morimoto, T. Soyama, Y. Hasegawa, K. Nakamura, T. Yamanishi, M. Sato and Y. Nakajima, Jpn. J. Radiol., 2014, 32, 242-250.

11 Y. Otani, M. Komura, H. Komura, T. Ishimaru, K. Konishi, H. Komuro, K. Hoshi, T. Takato, Y. Tabata and T. Iwanaka, Tissue Eng., Part A, 2015, 21, 627-636.

12 G. Li, M. Che, K. Zhang, L. Qin, Y. Zhang, R. Chen, L. Rong, S. Liu, Y. Ding, H. Shen, S. Long, J. Wu, E. Ling and Y. Zeng, Biomaterials, 2016, 83, 233-248.

13 R. Rohanizadeh, M. V. Swain and R. S. Mason, J. Mater. Sci.: Mater. Med., 2008, 19, 1173-1182.

14 S. Stanton, V. Salih, G. Bentley and S. Downes, J. Mater. Sci.: Mater. Med., 1995, 6, 739-744.

15 N. J. Goodstone, A. Cartwright and B. Ashton, Tissue Eng., 2004, 10, 621-631. 
16 M. Watanabe, J. Jo, A. Radu, M. Kaneko, Y. Tabata and A. W. Flake, Tissue Eng., Part A, 2010, 16, 1645-1655.

17 D. Zou, Z. Zhang, J. He, K. Zhang, D. Ye, W. Han, J. Zhou, Y. Wang, Q. Li, X. Liu, X. Zhang, S. Wang, J. Hu, C. Zhu, W. Zhang, Y. Zhou, H. Fu, Y. Huang and X. Jiang, Biomaterials, 2012, 33, 2097-2108.

18 M. Ou, Y. Zhao, F. Zhang and X. Huang, Connect. Tissue Res., 2015, 56, 204-211.

19 M. Pelaez, C. Susin, J. Lee, T. Fiorini, F. C. Bisch, D. R. Dixon, J. C. McPherson III, A. N. Buxton and U. M. E. Wikesjö, J. Clin. Periodontol., 2014, 41, 827-836.

20 K. Tsuji, A. Bandyopadhyay, B. D. Harfe, K. Cox, S. Kakar, L. Gerstenfeld, T. Einhorn, C. J. Tabin and V. Rosen, Nat. Genet., 2006, 38, 1424-1429.

21 A. W. James, G. LaChaud, J. Shen, G. Asatrian, V. Nguyen, X. Zhang, K. Ting and C. Soo, Tissue Eng., Part B, 2016, 22, 284-297.

22 H. Begam, S. K. Nandi, B. Kundu and A. Chanda, Mater. Sci. Eng., C, 2017, 70, 856-869.

23 M. Geiger, R. H. Li and W. Friess, Adv. Drug Delivery Rev., 2003, 55, 1613-1629.

24 C. H. Lu, Y. H. Chang, S. Y. Lin, K. C. Li and Y. C. Hu, Biotechnol. Adv., 2013, 31, 1695-1706.

25 H. Jin, K. Zhang, C. Y. Qiao, A. Yuan, D. Li, L. Zhao, C. Shi, X. Xu, S. Ni, C. Zheng, X. Liu, B. Yang and H. Sun, Int. J. Nanomed., 2014, 9, 2179-2190.

26 C. Cam and T. Segura, Curr. Opin. Biotechnol., 2013, 24, 855863.

27 S. Raisin, E. Belamie and M. Morille, Biomaterials, 2016, 104, 223-237.

28 J. H. Yu, J. S. Quan, J. Huang, C. Y. Wang, B. Sun, J. W. Nah, M. H. Cho and C. S. Cho, Acta Biomater., 2009, 5, 2485-2494.

29 E. S. Elsubeihi and J. N. M. Heersche, Arch. Oral Biol., 2004, 49, 401-412.

30 A. Kumasaka, M. Iikubo, T. Nishioka, I. Kojima, N. Shoji, M. Sakamoto and T. Sasano, Clin. Implant Dent. Relat. Res., 2015, 17, 1174-1179.
31 H. C. Liu, L.-L. E, D. S. Wang, F. Su, X. Wu, Z. P. Shi, Y. Lv and J. Z. Wang, Tissue Eng., Part A, 2011, 17, 2417-2433.

32 M. Endo, S. Kuroda, H. Kondo, Y. Maruoka, K. Ohya and S. Kasugai, Tissue Eng., 2006, 12, 489-497.

33 C. A. Dunn, Q. Jin, M. Taba Jr, R. T. Franceschi, R. Bruce Rutherford and W. V. Giannobile, Mol. Ther., 2005, 11, 294-299.

34 Y. Yang, F. A. Nunes, K. Berencsi, E. E. Furth, E. Gönczöl and J. M. Wilson, Proc. Natl. Acad. Sci. U. S. A., 1994, 91, 44074411.

35 J. Xu, Z. Li, Y. Hou and W. Fang, Am. J. Transl. Res., 2015, 7, 2527-2535.

36 N. Zhou, Q. Li, X. Lin, N. Hu, J. Y. Liao, L. B. Lin, C. Zhao, Z. M. Hu, X. Liang, W. Xu, H. Chen and W. Huang, Cell Tissue Res., 2016, 366, 101-111.

37 M. Bruderer, R. G. Richards, M. Alini and M. J. Stoddart, Eur. Cells Mater., 2014, 28, 269-286.

38 A. R. Shrivats, E. Hsu, S. Averick, M. Klimak, A. C. S. Watt, M. DeMaio, K. Matyjaszewski and J. O. Hollinger, Clin. Orthop. Relat. Res., 2015, 473, 2139-2149.

39 L. Peng, X. Cheng, R. Zhuo, J. Lan, Y. Wang, B. Shi and S. Li, J. Biomed. Mater. Res., Part A, 2009, 90, 564-576.

40 Y. Takahashi, M. Yamamoto and Y. Tabata, Biomaterials, 2005, 26, 4856-4865.

41 S. Hoshi, T. Akizuki, T. Matsuura, T. Ikawa, A. Kinoshita, S. Oda, Y. Tabata, M. Matsui and Y. Izumi, J. Periodontal Res., 2016, 51, 77-85.

42 V. Rosen and R. S. Thies, Trends Genet., 1992, 8, 97-102.

43 K. Ting, L. A. Petropulos, M. Iwatsuki and I. Nishimura, J. Bone Miner. Res., 1993, 8, 1377-1387.

44 G. N. King, N. King, A. T. Cruchley, J. M. Wozney and F. J. Hughes, J. Dent. Res., 1997, 76, 1460-1470.

45 K. Matin, H. Nakamura, K. Irie, H. Ozawa and S. Ejiri, Int. J. Oral Maxillofac. Implant., 2001, 16, 400-411.

46 C. W. Müller, K. Hildebrandt, T. Gerich, C. Krettek, M. van Griensven and E. Rosado Balmayor, J. Tissue Eng. Regener. Med., 2015, 11, 1122-1131. 